\title{
TurfNet: An Architecture for Dynamically Composable Networks
}

\author{
Stefan Schmid, Lars Eggert, Marcus Brunner, and Jürgen Quittek \\ NEC Europe Ltd., \\ Network Laboratories, \\ Kurfürstenanlage 36, \\ 69115 Heidelberg, Germany \\ \{schmid, eggert, brunner, quittek\}@netlab.nec.de
}

\begin{abstract}
The Internet architecture is based on design principles such as endto-end addressing and global routeability. It suits relatively static, well-managed and flat network hierarchies. Recent years have shown, however, that the Internet is evolving beyond what the current architecture can support. The Internet architecture struggles to support increasingly conflicting requirements from groups with competing interests, such as network, content and application service providers, or end-users of fixed, mobile and ad hoc access networks. This paper describes a new internetworking architecture, called TurfNet. It provides autonomy for individual network domains, or Turfs, through a novel interdomain communication mechanism that does not require global network addressing or a common network protocol. By minimizing inter-domain dependencies, TurfNet provides a high degree of independence, which in turn facilitates autonomic communications. Allowing network domains to fully operate in isolation maximizes the scope of autonomic management functions. To accomplish this, TurfNet integrates the emerging concept of dynamic network composition with other recent architectural concepts such as decoupling locators from identifiers and establishing end-to-end communication across heterogeneous domains.
\end{abstract}

\section{Introduction}

The Internet has evolved from a small research network to a huge, worldwide information exchange that plays a central role in today's societies. A growing diversity of interests in this global internetwork (e.g., commercial, social, ethnic, governmental, etc.) leads to increasingly conflicting requirements among competing stakeholders. These conflicts create tensions the original Internet architecture struggles to withstand.

As one example of an ongoing "tussle" [1], consider the commercial success of the Internet. It has created a large number of competing service providers that aim to outperform one another in order to increase their profits. The result is an increased willingness to forgo agreed-upon standards that allowed a more cooperatively managed Internet to succeed. This paper argues that despite the remarkable success of the Internet architecture - often attributed to its robust design principles - its underlying 
assumptions no longer fully match today's networking requirements. In particular, specialized new types of networks, such as sensor networks, mobile ad hoc networks and the widespread deployment of "middleboxes" have begun to stretch the capabilities of the existing architecture. This has prompted research into fundamentally different network architectures, such as FARA [2], Plutarch [3], Triad [4] or IPNL [5].

This paper proposes a new internetworking architecture called TurfNet, which addresses the limitations of the Internet architecture by accommodating conflicts of interests among different stakeholders and supporting their diverse interests.

The TurfNet architecture focuses on interoperation between otherwise autonomous networks. These autonomous networks are modularized according to the inherent boundaries drawn by the different interests of the stakeholder involved. This paper uses the name turf to denote such an autonomous network. The term turf has an innate connotation to ownership and responsibility that the TurfNet architecture reflects. Other papers introduce different terms for similar concepts, such as regions [6] or contexts [3]. The concept is also related to the Internet's Autonomous Systems (AS).

Isolated, autonomous TurfNets dynamically compose into new, larger autonomous TurfNets that integrate the original networks. The process of dynamic network composition supports the interconnection of heterogeneous networks, such as mobile and ad hoc networks, IPv4 networks or IPv6 networks. Composed "super" networks manage this integration by abstracting potential isolation (e.g., over-lapping address spaces) or heterogeneity (e.g., incompatible network protocols) issues among the constituent subnetworks. One mechanism for supporting this heterogeneity is address and protocol translation, but the architecture supports other, equivalent mechanisms as well.

Backwards compatibility with today's Internet is a crucial requirement for any next-generation internetworking architecture. This was arguably one critical mistake during the design of IPv6. The TurfNet architecture maintains compatibility with the current Internet architecture by supporting it as one specific network type, along with 3G mobile networks, ad hoc networks or sensor nets.

The first part of this paper motivates this research and discusses the underlying design principles of the architecture. Section 3 then outlines the TurfNet architecture and explains how it addresses the "new" needs of today's networking requirements. Section 4 describes the basic end-to-end communication across several layers of composed TurfNets. Section 5 then discusses the scalability properties of the TurfNet architecture. Finally, the remaining sections of this paper compare and contrast the TurfNet architecture against other related work and conclude with an outlook on future work.

\section{Design Axioms}

This section briefly discusses the basic axioms of the TurfNet architecture.

Packet switching. Packet-switched networks increase performance and efficiency by multiplexing bursty traffic from different sources onto the same medium. Furthermore, packet switching provides a simple, generic communication framework that 
supports many different kinds of data flows and requires little explicitly managed state inside the network.

Separation of identity and location. Today's IP addresses denote both the identity of a node as well as its topological location. Several proposals for splitting the two functionalities exist, and the TurfNet architecture will adopt this important new concept with a focus on supporting mobility.

Global namespace. The Internet's Domain Name System (DNS) [7] is a global, hierarchical namespace based on Fully Qualified Domain Names (FQDNs). Other current naming schemes, such as the ones used for the Host Identity Protocol (HIP) [8] or the Layered Naming Architecture [9], also make use of globally unique names or identities.

Similar to these approaches, the TurfNet architecture globally identifies network entities belonging to different, autonomous TurfNets. Consequently, the TurfNet architecture could either use FQDNs, HIP identities, or any other global namespace - or even different global namespaces at the same time. For simplicity, the remainder of this paper uses the generic term "name" to refer to arbitrary types of global identifiers.

Flexibility in business models. Networking moves from a few monolithic operators to a scenario where the competing interests of owners, roaming brokers, transit network operators, users, and service providers, among others, must be accommodated and balanced. Consequently, a future internetworking architecture must enable, support and manage new business models and complex value chains.

Autonomous Turfs. Braden [10] proposes the meta-architectural principle that different regions of the network should be allowed to differ from each other: "minimize the degree of required global architectural consistency." This paper adopts this principle as a necessary enabler for future businesses and diversity between domains.

Inter-Turf control interface. Network control must cross domain boundaries, for example, to support address registration and name lookups across individual TurfNets. Such functions require a common, high-level inter-Turf control interface to exchange control state and configuration information. This facility must not be tied to a specific network protocol - which could be different within individual TurfNets - but rather depend on a common data format.

\section{The TurfNet Architecture}

A TurfNet is a completely autonomous network domain. To achieve autonomy, every TurfNet encompasses its own, independent network addressing mechanism and all associated control plane functions, such as routing protocols, name-to-address resolution, etc. A common, shared namespace to enable inter-Turf communication is the only global requirement (apart from the high-level inter-Turf control interface). In contrast to today's Internet architecture, TurfNets do not rely on globally shared state or pervasive functionality like a common network protocol, a globally shared address space or a global name service. 
Another fundamental design choice that supports autonomy of TurfNets is the concept of encapsulation. It allows TurfNets to fully hide their internal characteristics, structures and policies. Such a modular network architecture allows individual players with potentially competing interests to interoperate in a controlled and protected manner and thus better suites the new requirements of future network communication.

If a TurfNet chooses to hide its internals (e.g., network addresses and protocols), external nodes cannot directly communicate with individual nodes of that TurfNet anymore. Communication without knowledge of the peer node's local network address (and protocol) requires new network capabilities. In the TurfNet architecture, nodes first have to acquire a Turf-local representation in the destination TurfNet. In essence, each TurfNet maps the remote communication peers into part of its local address space. To other local TurfNodes, remote nodes appear to be of the local Turf.

\subsection{Architecture Overview}

Figure 1 shows an abstract view of the proposed TurfNet architecture. Its key components are:

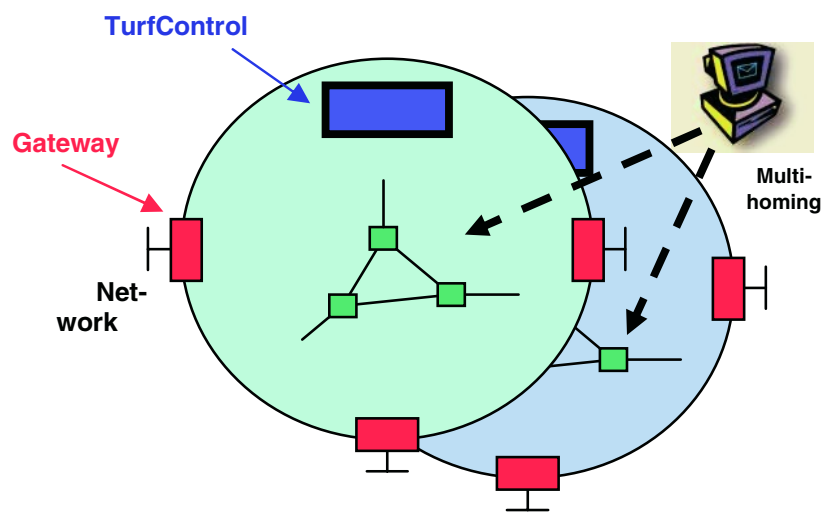

Fig. 1. The TurfNet Architecture

TurfControl. The TurfControl is a logical, per-Turf entity comprised of a TurfNet's essential control functions and services. It encompasses all traditional control plane functionalities in the network, such as address allocation, routing and name resolution. It further includes the new TurfNet functionality, for example, to manage TurfNet composition.

A TurfNet handles all its control functionality locally. This is an important prerequisite for maintaining the autonomy of individual TurfNets. Because of the importance of the TurfControl for the proper operation of a TurfNet, it must be resilient. In the case of large (composed) TurfNets, distribution and replication of this logical functionality across many nodes will improve scalability as well as resilience.

TurfNode. A TurfNode is a network node in a specific TurfNet. It communicates with Turf-local network protocols and uses local addressing and routing mechanisms. 
A TurfNode interacts with the local TurfControl for all control plane operations, such as address allocation, routing or name resolution.

To support multi-homing as a fundamental part of the TurfNet architecture, physical nodes may concurrently participate as full-fledged logical TurfNodes in multiple TurfNets (see Figure 1). Note that multi-homed nodes do not necessarily act as gateways between the different TurfNets (see below).

Gateways. TurfNet gateways are special, multi-homed nodes. Besides being part of multiple Turfs at the same time, they also actively relay traffic between the different TurfNets they are a part of. To enable this functionality, such gateways must be fully operational TurfNodes in both TurfNets (i.e., they must be able to communicate and have at least one interface in both TurfNets).

The main responsibility of these gateway nodes is to relay traffic between the different TurfNets. In the case of peering, TurfNets use independent network addressing or even different network protocols. Gateways will then also perform the required address and protocol translations. For example, a gateway between IPv4 and IPv6 TurfNets will translate between the two network protocols and their respective address spaces. If two TurfNets use the same protocols and have compatible addressing, the gateway will simply forward data packets - acting like a traditional Internet router. Whether a Turf gateway acts as a traditional router, as network address translator, or even as protocol translator depends on its local environment. Section 4 discusses advantages and disadvantages of the different roles further.

Another task of gateways is to connect the TurfControls of the peering TurfNets. If TurfNets use different control protocols, the gateway must translate control messages.

\subsection{Network Composition}

The TurfNet architecture adopts the central architectural principle of network composition from the Ambient Networks project [11]. In this context, network composition is introduced as a new paradigm that allows co-operative networks to automatically negotiate inter-working agreements through which they establish inter-domain communication. How network composition at the control plane level can facilitate selforganisation is discussed in a related paper [12] by Kappler et al. In the context of this work, on the contrary, network composition is considered a means to inter-connect fully autonomous networks (for example, TurfNets), whereby the focus lays on interdomain communication among heterogeneous networks (including different network protocols and address spaces).

TurfNets can dynamically compose with each other to form new, integrated or interconnected TurfNets. Two different variants of this operation are possible, resulting in horizontal or vertical composition of individual networks.

\subsection{Horizontal Composition}

When multiple TurfNets merge into a single TurfNet such that they share a common control plane as a result, they compose horizontally. This type of composition fully integrates the original TurfNets into the final, merged TurfNet. For example, one TurfNet could adopt the addressing mechanisms and protocols of the others, or the 
merging TurfNets could all agree on a new set of addressing mechanisms and protocols. Figure 2 illustrates this process.

A key characteristic of horizontal composition is that merged TurfNets have a single logical TurfControl instance. The original TurfNets lose their "identity" after the composition and appear from then on only as part of the identity of the new TurfNet. The process of horizontal composition is irreversible - no information about the original constituents exists that would allow decomposition into the original TurfNets.

Despite this loss of identity, horizontally composed networks can still split. This occurs, for example, when a TurfNet becomes partitioned due to network link failures. However, partitioning will not typically restore the original TurfNets but instead result in an arbitrary set of TurfNets.

Finally, note that the TurfNet architecture does not prohibit a TurfNet from being structured into different administrative domains. These domains, however, are management entities that are not visible in an architectural description of a TurfNet.

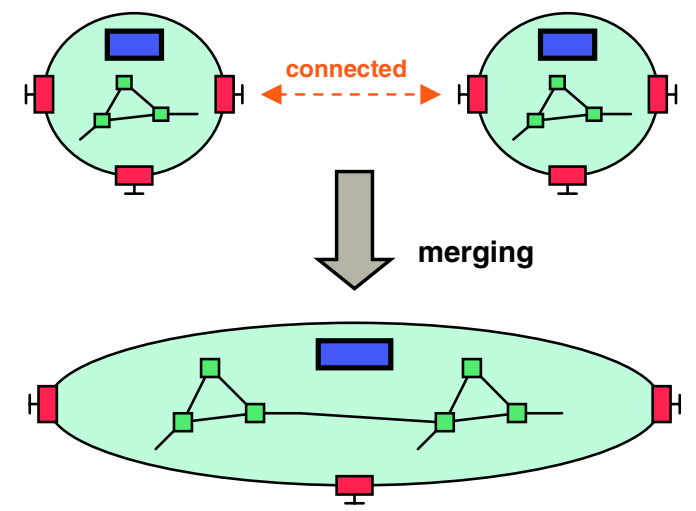

Fig. 2. Horizontal composition of TurfNets

\subsection{Vertical Composition}

Vertical composition, on the other hand, is the process by which TurfNets compose such that each individual TurfNet preserves its autonomy (with respect to addressing, routing, name resolution, etc.) even after it becomes part of the newly composed TurfNet. In this case, two TurfNets are said to compose vertically such that one becomes the governing parent TurfNet of the other. Figure 3 illustrates this case and Figure 4 highlights hierarchical structure of this composition variant.

The advantage of vertical network composition is encapsulation of administrative, control and routing functionalities, as well as isolation of internal structures. Because of hierarchical composition, new sub-TurfNets may join locally, without requiring global interaction. This reduces the complexity of administrative and control negotiations. 


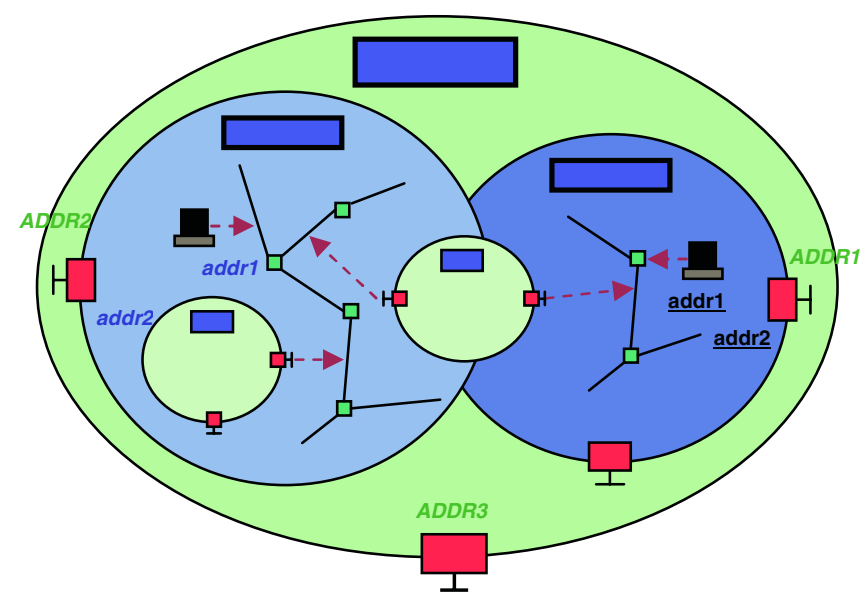

Fig. 3. Vertical composition of TurfNets

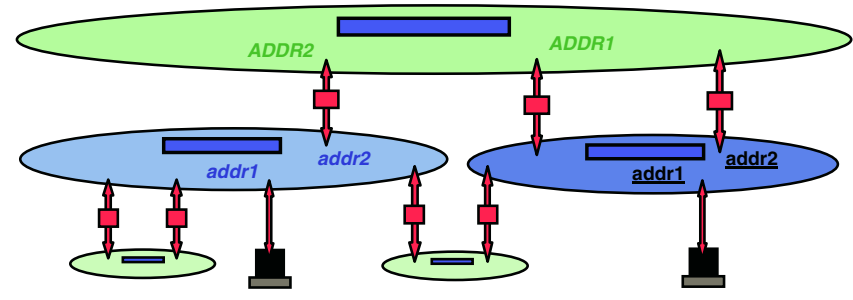

Fig. 4. Hierarchically structured TurfNets after vertical composition

Similar to the proposed TurfNet architecture, the Internet also contains administrative domains that hide the internal complexity of a domain (e.g., intra-domain routing). However, the main difference to the TurfNet architecture is that its vertical composition is not limited to two levels. Furthermore, vertical composition fully separates administrative and control functionalities (i.e., not even the addressing or routing must be globally agreed upon).

Another important advantage of vertical composition is that the transition from today's networking infrastructure requires few changes to existing networks. Specialized gateway nodes facilitate interoperability and integration by translating between different domains. For example, vertical composition can integrate existing IPv4 and IPv6 networks without modifications to existing protocols and protocol stacks.

Communication across vertically composed TurfNet boundaries occurs through well-defined gateways, which relay traffic between the different TurfNets. If the peering TurfNets use the same network protocol and non-overlapping network addresses, a gateway simply forwards packets between the domains, similar to traditional Internet routers. However, if TurfNets use different network addressing schemes or different network protocols, a gateway also performs bidirectional network address and protocol translation. 
The number of gateways used to compose a TurfNet into a parent TurfNet depends on reliability and scalability requirements. Large volumes of traffic require a larger number of gateway nodes, to share the processing load of address translation. Multiple gateways also provide resilience in the case of gateway failures.

As illustrated in Figure 4, TurfNets can simultaneously compose with several higher-layer TurfNets. This is especially important for multi-homed TurfNets that peer with several providers.

\subsection{Discussion}

The previous, brief description of composition approaches has illustrated that horizontal and vertical composition are fundamentally different and address different architectural needs.

Horizontal composition fully integrates two networks into one. In this case, composition only occurs during the initial setup phase of the network integration - afterwards, the composed TurfNet operates exactly as a monolithic TurfNet would. In other words, composition does not visibly affect performance, scalability, security or other network properties.

Horizontal composition of networks allows integration of networks belonging to the same administration or different administrations. However, horizontally composing networks must all agree on the same address space, address allocation scheme and require a common routing mechanism. One example of horizontal composition occurs between different networks of a single or multiple cooperating network providers. However, composition between a service provider network and its customers' networks requires a different type of composition due to lack of trust and the desire to preserve some level of autonomy between the different parties. Consequently, a more loosely coupled form of composition is needed.

Vertical composition provides this looser form of composition. It enables independent networks with different network architectures that may belong to separate administrations to compose in a way that preserves their individual autonomy and specific internal operation. The gateway nodes, which are configured through the TurfControls involved in the composition process, enable the integration and interoperation of the otherwise fully independent networks. The overhead associated with this loose type of composition is acceptable in cases where closer composition is not an option due to administrative concerns, e.g., lack of trust or desire for autonomy).

In terms of the TurfNet architecture, the existing Internet topology can be interpreted as consisting of horizontally composed networks, each being its own administrative domain or autonomous system. They share a common address space together with common routing and name resolution functions. The TurfNet architecture enables composition of this Internet-wide TurfNet with new access networks that feature different control functions, or for example, with vehicle area networks (VANs) that use their own non-IP communication infrastructure, Bluetooth-based personal area networks (PANs), or ad hoc networks. All of these networks temporarily or permanently compose with the current Internet through TurfNet gateways. The only constraint is that a common, global name space for all of them needs to be place. 
Due to space limitations, the remainder of this paper focuses on the - arguably more interesting - vertical TurfNet composition, even though both types of composition are equally important pieces of the overall architecture.

\section{Basic Operation}

End-to-end communication across TurfNet boundaries is not trivial due to isolation and heterogeneity of the individual networks. TurfNet adopts the decoupling of names and locators from FARA's abstract architectural model [2]. It therefore uses names as global identifiers of TurfNodes that are different from the node addresses used for routing.

Because TurfNode addresses may not have end-to-end significance (they might merely be transient routing tags for Turf-local routing), the architecture uses the name registration and resolution process to find and setup the high-level routing path across composed TurfNets. End-to-end communication across TurfNet boundaries is thus a product of the following processes: address allocation, node registration, name resolution and packet relaying.

\subsection{Turf-Local Address Allocation}

A TurfNode joining a TurfNet first needs to obtain a Turf-local network address using the respective address allocation function of the TurfControl (e.g., DHCP [13] or IPv6 auto-configuration [14]). This address only needs to be valid and meaningful within the local Turf. How individual TurfNets handle address assignment does not affect the inter-Turf architecture. Ideally, this process of address allocation should happen in a fully automated way.

\subsection{Node Registration}

Because individual TurfNets may have completely independent network addresses spaces, TurfNodes may not be directly addressable from outside their local Turf (similar to today's NAT'ed hosts). The lack of a global address space across all TurfNets prevents an external node (of another TurfNet) from addressing a local node directly. The TurfNet architecture exacerbates this problem, because different TurfNets may not only have overlapping but also completely different address spaces or network protocols (e.g., IPv4, IPv6, or any other internetworking protocol).

Although NATs are often held responsible for breaking the end-to-end semantics of the Internet - and rightfully so - their hiding capabilities are a central feature of the TurfNet architecture. However, TurfNet carefully eliminates the disadvantages of NATs by introducing names as explicit, end-to-end node identifiers. Gateways are free to translate addresses and protocols when higher-layer entities bind to static names. Hiding TurfNet internals facilitates strong autonomy between TurfNets and minimizes shared global state. The ability to hide the internals of networks and the resulting autonomy is becoming critically important in today's commercial Internet, where even companies that own sufficient address space use NATs to control outside visibility of internals of their local networks. 
A TurfNode that wants to be reachable for nodes outside of its local Turf registers its local address with the name resolution service of the local TurfControl (step 1 in Figure 5). To achieve reachability from external TurfNodes, this registration propagates up the hierarchy of composed TurfNets (step 2) via the TurfControl of the local TurfNet. Note that a TurfNode itself cannot register its address in external TurfNets, because it is not directly aware of their existence.

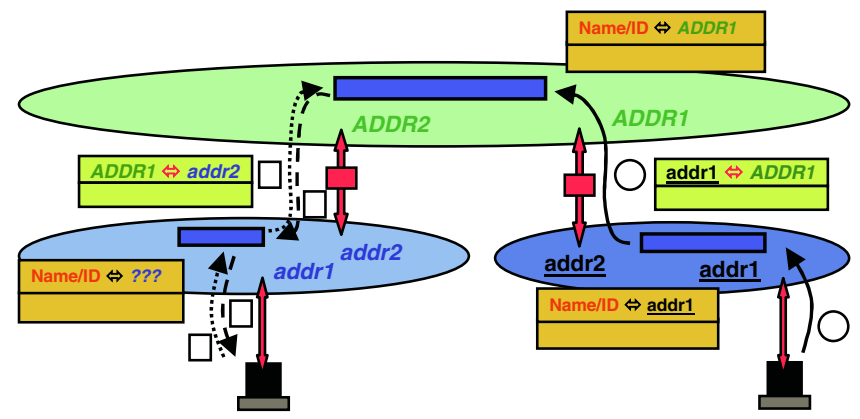

Fig. 5. Node registration and name resolution across TurfNets with independent address spaces

If a child and a parent TurfNet use separate or even different address spaces, a straightforward propagation of the address registration to the parent TurfNet would fail, because the addresses of the registered TurfNode have no meaning outside its local TurfNet. Consequently, a new proxy address for such a node needs to be allocated and registered with the parent TurfNet. This process may cause a node to receive different local proxy addresses in each TurfNet along the path. The gateways initiate the allocation of proxy addresses. For each address registration propagated to a parent TurfControl, the gateway that connects the TurfNets requests a new local network address from the address allocation function of the parent TurfControl. It then creates the necessary address/protocol translation state between the different addresses. The address allocation function operates in a distributed fashion; for example, each gateway might control its own pool of addresses.

Mappings from names to proxy addresses are soft state that times out if not refreshed periodically. This conforms to the requirement of using soft state between Turf boundaries. Using soft state for address mappings can also significantly reduce the necessary state in the gateways, because they maintain only the state associated with active nodes at any given time.

\subsection{Name Resolution}

If a TurfNode wants to communicate with a peer node, it requests name resolution through the Turf-local name resolution service. If the peer node is part of the same TurfNet, this is a fully local operation, as illustrated by steps 3 and 6 in Figure 5.

However, if the peer node is not part of the local TurfNet, the local name resolution function propagates the lookup to its parent $\operatorname{TurfNet}(s)$, which then try to resolve the 
name within their respective domains (step 4). Because addresses may only be valid within a single Turf, the addresses that name resolution returns may differ from TurfNet to TurfNet. Thus, if the child and parent TurfNet use a different address spaces, gateways must allocate a new proxy address for the resolved name after a successful name resolution by a parent TurfNet.

When gateways must allocate a local proxy address (because the child and parent TurfNets use different address spaces), they also install the necessary address/protocol translation state for mapping between the different address spaces and/or network protocols. The gateway that receives the successful reply from the parent TurfNet can either perform this operation "in-band" or the local TurfControl can perform this operation "out-of-band" after it receives the name resolution response.

This address/protocol translation state is also soft state, in order to reduce gateway state when only a few inter-Turf communications are active. The specific conditions for flushing soft state depend on the individual types of communication. For example, exceeding the maximum idle time of an address translation entry could invalidate the soft state mapping between proxy addresses names.

It is a characteristic of the TurfNet architecture that both address registration and name resolution may include address allocation and creation of address/protocol translation state.

\subsection{Packet Relaying}

End-to-end communication among TurfNodes can begin as soon as the address resolution process completes successfully. If both communicating peers belong to the same TurfNet, their communication is a completely local process that does not involve inter-Turf mechanisms.

If the communicating peers belong to different TurfNets, packet relaying involves the following steps.

First, if the peering TurfNets use the same network address space and communication protocols, the gateway nodes merely act as traditional routers and forward traffic between the different administrative domains. The Turf-local routing protocols then connect (across the Turf boundaries) to facilitate inter-Turf routing in much the same way as today's inter-domain routing protocols do (e.g., BGP).

Second, if the peering TurfNets use independent address spaces and/or different network protocols, Turf gateways also perform the necessary address and protocol translations when relaying packets. In this case, the address registration and name resolution procedures have already established the necessary proxy addresses and network address translation state at the gateway nodes along the communication path. Similar to today's Internet NATs, a TurfNet gateway adds a dynamic address translation rule for the reverse direction when a local TurfNode initiates communication with an external node. For this to work, a TurfNet gateway has to maintain network address translation state in both directions and must perform address translation on both source and destination addresses. This is often referred to as twice-NAT [15]. 
The result is that communication across TurfNets must always follow symmetric paths, because only those gateways have the necessary translation state. This is a change from the current Internet, which supports unidirectional links that cause forward and reverse traffic between two nodes to follow different, asymmetric paths. In reality, however, many Internet protocols and services do not deal with asymmetric paths well, especially if their characteristics (e.g., bandwidth or delay) are sufficiently different. In the future, asymmetric paths across TurfNets could be supported by coordinating translation state across different sets of gateways for the forward and reverse path. The management and security aspects of such approaches are currently not well understood and the present TurfNet architecture consequently limits itself to supporting symmetric paths only.

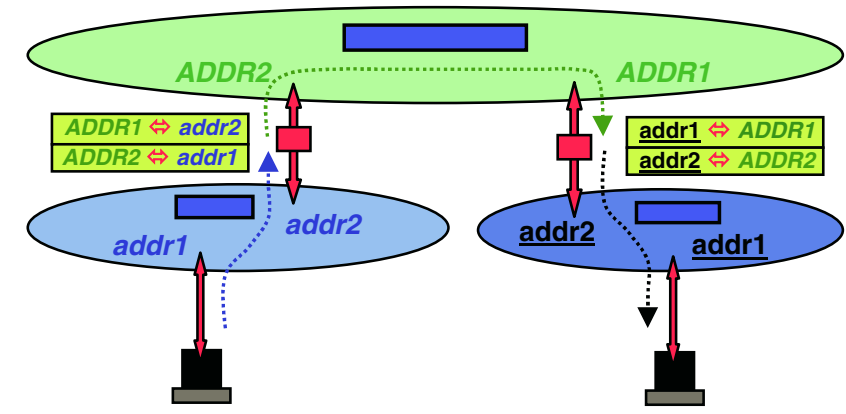

Fig. 6. NAT-based packet relaying in case of peering TurfNets with independent address spaces and/or protocols

Figure 6 illustrates packet relaying in detail. Note that although the example shown there is a simple two-stage scenario, the basic mechanism extends recursively to multiple stages. In the left of Figure 6, the initiator of the communication has address addr1 obtains an external proxy address $\boldsymbol{A D D R} 2$ in its parent TurfNet and creates an entry in the address translation table that maps between those addresses: $A D D R 2 \Leftrightarrow$ addr1. Note that different fonts are used for different address spaces: lower-case letters for the lower left, underlined letters for the lower right, and uppercase letters for the top TurfNet.

Figure 6 also illustrates the establishment of dynamic address translation state at the TurfNet gateways for reverse communication. Each gateway along the path must translate the source address of the sender. For this to work, these gateways must also establish a proxy address for the sender in every TurfNet along the transmission path. For the example in Figure 6, this means that the gateways first translate the sender's address (addr1) into proxy address $\boldsymbol{A D D R} 2$ at the top-level TurfNet and then into addr2 at the receiver's TurfNet.

When this stage finishes, it has created all required proxy addresses and their corresponding address translation state. Bidirectional communication between the peers is now possible through straightforward address translations. 


\section{Scalability Considerations}

One central assumption of the TurfNet architecture is that no hard state exists between individual TurfNets; consequently, gateways and name resolution services may only use soft state. Especially critical for scalability and performance is the efficient storage of name-to-address bindings and network address translation state. Because the TurfNet architecture decouples domains, each TurfNet is free to choose an appropriate solution that satisfies its particular scalability and performance needs.

\subsection{Namespaces and Name Resolution}

One of the central ideas of the TurfNet architecture is the complete autonomy of individual TurfNets. They do not require global state, globally unique names or global identities for all communication entities. However, to reduce the problem of global naming to the problem of a global namespace (rather than to a globally distributed name service as in today's Internet), every TurfNet provides a local name resolver as a basic functionality of the TurfControl. The problem of assigning globally unique names is an orthogonal issue to be solved outside the TurfNet architecture.

Local name resolvers in every TurfNet that map globally unique names to Turflocal addresses allow each TurfNet to operate completely autonomous, without the need of external naming services.

In contrast to the Internet's Domain Name Service (DNS), name-to-address mappings in TurfNet are soft state, i.e., they need to be updated regularly or they disappear automatically. Therefore, each (name, address) tuple has an associated lifetime. A TurfNode registers its Turf-local network addresses with the name service and must then periodically refresh these registrations to prevent them from timing out. Furthermore, (name, address) tuples have additional cache timers that are similar in function to DNS timers. They indicate how long clients may cache name-to-address resolutions before they must refresh them. A cache timer of zero indicates that clients must perform a new name resolution for each new transport-layer connection. For example, a cache time of zero can be used for mobile nodes that frequently change location.

Figure 5 illustrates inter-TurfNet communication, which relies on the fact that local TurfNodes that decide to be reachable from remote TurfNodes register their location with their parent TurfNets. This hierarchical name registration and resolution process ensures that a TurfNode that is part of any TurfNet in the overall composition can locate any registered node. If the Turf-local name service cannot resolve the address itself, the lookup request recursively propagates to the parent TurfNets until the name is resolved. Again, this recursive propagation is somewhat similar to the DNS.

A negative side effect of hierarchical name resolution is that top-level TurfNets require name-to-address mappings for all registered hosts, i.e., all hosts that choose to be reachable by any node in the composed TurfNets. For example, if the Internet were to be rebuilt from composed TurfNets, the top-level TurfNet would require (name, address) tuples for any host that wants to be globally reachable. This example shows the importance of scalability considerations for large (composed) TurfNets. 
An obvious approach to address the scalability issues is distributing the name-toaddress resolution service across many servers. This approach can achieve a high degree of load balancing and fault tolerance. For example, a distributed hash table, such as Chord [16], FPN [17] or Koorde [18], could provide the necessary levels of fault-tolerance and performance for very large numbers of nodes.

The scalability of a name resolver depends entirely on the potential size of its TurfNet and the number of nodes that decide to be globally reachable. For example, small TurfNets such as an ad hoc access network or a Personal Area Networks (PAN) may simply implement a centralized resolver.

The TurfNet architecture hides the internal structures and control functions of TurfNets and consequently leaves the implementation characteristics of these services to the individual TurfNets. For example, whereas a personal-area TurfNet may implement the TurfControl as a centralized service on a single node, a larger composed TurfNet that potentially encompasses a worldwide network with billions of hosts must obviously choose a very different implementation approach to fulfill its specific scalability and performance demands.

\subsection{Name Resolution Delegation}

This section outlines a particular name resolution mechanism that supports the specific requirements of the TurfNet architecture and can scale up to large composed TurfNets with billions of TurfNodes. The proposed name resolution mechanism is of special interest for the TurfNet architecture as it supports highly dynamic address updates despite large-scale deployments (as for example needed for future mobile networks).

The main idea of the proposed mechanism is to split the name resolution process into two steps. The first step simply resolves the Delegate Address Resolver (DlgAR), which is then responsible for the actual name-to-address resolution in a second step (see Figure 7).

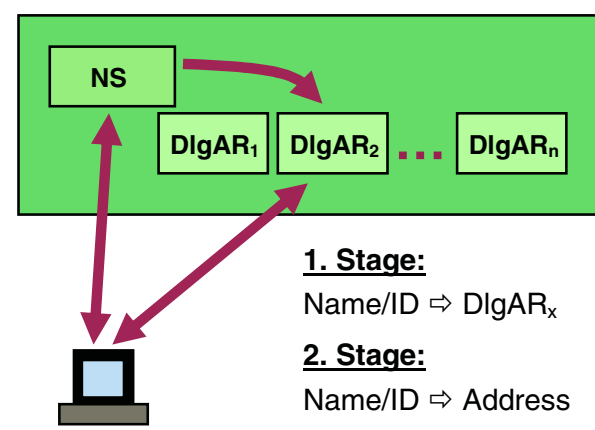

Fig. 7. Delegate name resolution system

Because the mapping from an actual name (or host identity) to the responsible $D \lg A R$ is expected to be relatively static, the result of this resolution is cacheable for 
long durations. For example, if this first mapping is based on the name (or host identity) prefix (e.g., all names starting with "a..." map to $D \lg A R_{x}$, “www.ab..." to $D \lg A R_{y}$, etc.), repetition of the first lookup step will be very rarely needed. This achieves a high level of load balancing, because most lookups will only involve the responsible $D \lg A R$ s.

One benefit of this distribution mechanism is that only a single instance (or at the most a few instances, for reason of fault tolerance) maintains the actual name-toaddress mapping ${ }^{1}$. This enables highly dynamic changes, without the overhead of updating many servers. For example, a mobile node that changes its points of network attachment frequently could use this name resolution mechanism to handle the mobility management for new connections.

To further increase scalability of the proposed solution, one could extend the twostage name lookup mechanism into a multi-stage name lookup mechanism.

\subsection{Aggregation of Address Translation State in Gateways}

Besides the scalability concerns of name resolution systems in large (composed) TurfNets, the architecture also has stringent scalability requirements for gateway nodes. For gateways to allow inter-TurfNet communication, they must perform address translations on all inbound and outbound packets. For large (composed) TurfNets, this requires the gateways to maintain a dynamic proxy address for any registered or active host that uses it. Besides proxy addresses, the gateway also has to hold the necessary address and/or protocol translation state.

Because a gateway that connects to a top-level (composed) TurfNet may provide address translation functionality for potentially huge numbers of nodes, minimizing required state is important. The amount of state required at Turf gateways is expected to be of a similar order of magnitude as in today's NAT gateways. Therefore, translation state is not expected to be a limiting factor for scalability.

Nevertheless, state aggregation can minimize state in TurfNet gateways. The basic idea is for gateways to allocate dynamic proxy addresses for nodes of sub-TurfNets in a way that aggregates addresses, such that one or at the most a few separate entries exist in the address translation table.

The following example illustrates how TurfNets can aggregate state. Without loss of generality, the example uses familiar IPv4 addresses. In this example, addresses only have to be unique within a single TurfNet. Even sub-TurfNets can use overlapping addresses. Figure 8 illustrates state aggregation in the case of an IPv4-based addressing scheme. The top-level TurfNet gateway on the left maintains only two aggregated proxy addresses (namely, 10.1.0.0/24 and 10.2.0.0/16) and the relevant address translation state for those sub-TurfNets.

This example also shows the advantage of aggregation for the address translation process. In the case of aggregated addresses (for example, the second entry in the address translation table of the top-level gateway: 10.2.0.0/16 $\Leftrightarrow 10.10 .0 .0 / 16)$, the gateway only has to translate the prefix of the host addresses. In this particular example, only the class B prefix requires translation.

\footnotetext{
${ }^{1}$ Note that today's DNS, which achieves scalability through extensive caching and replication, is not suitable for mobile environments, where dynamic address changes are frequent.
} 


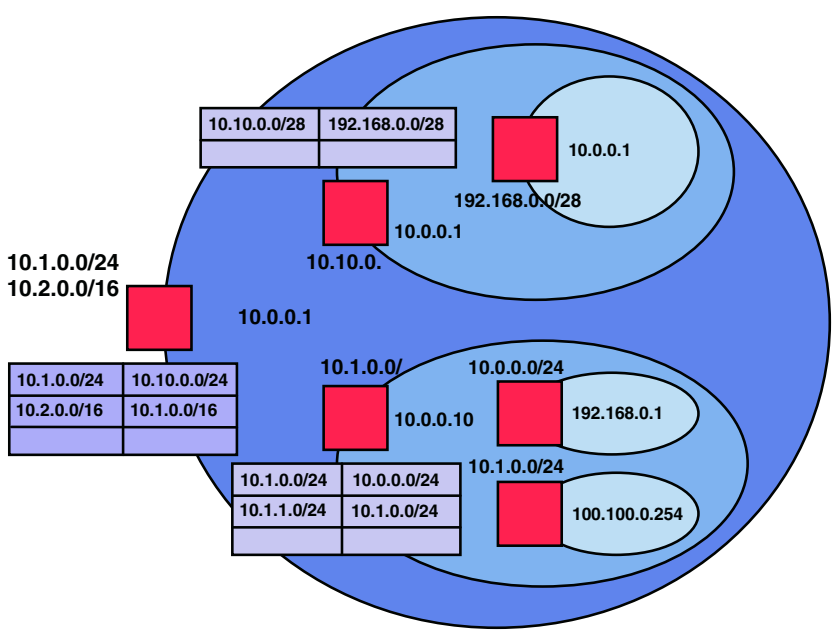

Fig. 8. Aggregation of address translation state

The prefix-based address translation method, based on subnet addresses rather than individual host addresses, has the advantage of only requiring a fraction of the regular address translation state. Prefix-based address translation can also reduce the processing overhead in gateway nodes, as only parts of the addresses must be changed. Particularly in gateway nodes of large composed networks, where address translation state may be highly aggregated, translations would affect only small parts of addresses.

\section{Architectural Evaluation}

This section evaluates the proposed TurfNet architecture through a qualitative examination of the following aspects:

Scalability. The main bottlenecks of the architecture regarding scalability are the explicitly defined gateway nodes that relay inter-TurfNet traffic. The relay method routing, address translation and/or protocol translation - can significantly affect TurfNet performance for large numbers of communication hosts and/or data flows. For example, when two core network providers compose their high-speed networks, performing address and/or protocol translation can be problematic. A better approach is to agree on a common address space and routing scheme. On the other hand, when a PAN network dynamically composes with a wireless hotspot network, composition of the different network types through network address translation may be an effective solution.

State aggregation mechanisms can mitigate the problem of state explosion in gateway nodes, as discussed above. However, another approach may be requires to address performance or load problems associated with address translation of all interTurfNet communication. One obvious approach is to introduce sufficient gateways to 
load-balance the necessary translation work. Note that the TurfNet architecture does not limit the number of gateways per TurfNet. Additional gateways will only add some extra control traffic.

Resilience. Inter-TurfNet communication relies on dedicated gateway nodes that are able to relay traffic between neighboring TurfNets. Hence, the architecture depends on the correct operation of those nodes. This dependency is similar to today's Internet, where NAT'ed networks also depend on the correct operation of the NAT gateway and the Internet at large depends on the correct operation of its routers.

One way to address this problem is to introduce sufficient backup gateways to allow failovers. However, designing a sufficiently fast failover mechanism may prove a challenge. Another approach to improve fault tolerance in the TurfNet architecture is through configuration of redundant gateway paths during the initial address registration phase and when resolving the addresses of a communication peer. Note that this could be done by the TurfControls along the inter-Turf path in a way that is completely transparent to the end nodes. The use of alternative addresses when establishing the end-to-end communication path to a TurfNode enables creation of disjoint paths. TurfControls along the inter-Turf end-to-end communication path recursively choose different gateways and thus disjoint high-level paths for the alternative addresses.

Because communication based on redundant peer addresses flows over a different set of gateway nodes, failures of one gateway on the original path will not affect the others. This allows the communication initiator to switch to a peer's alternative address (thus using alternative paths) in the presence of a gateway failure. The fact that alternative paths through different gateways likely pass through different network service providers further increases resilience, because this approach circumvents problems that could affect whole provider networks.

Performance. The impact of the proposed TurfNet architecture on the overall networking performance must be considered carefully. The fact that all inter-TurfNet traffic passes through fixed gateway nodes introduces several potential network bottlenecks.

Today's Internet, in some respects, suffers from the same problem, because many home and corporate networks are located behind NAT boxes. In addition, most 2.5/3G mobile access networks relay all external communication through static NAT gateways. Nevertheless, the fact that today's Internet operates - despite the large number of middleboxes - illustrates that performance problems due to NATs are solvable. Correct provisioning of the gateway nodes, both in terms of performance and numbers, is important with an increasing volume of inter-TurfNet communication.

One way to address potential performance problems of TurfNet gateways due to extensive address translations is dedicated hardware support. Existing hardware solutions for high-speed "label switching" systems (e.g., ATM, MPLS) could in the future also support fast address lookups and rewrites of source and destination addresses.

Flexibility. The key design objectives of the TurfNet architecture are creating administratively independent, autonomous networks domains and allowing their dynamic 
composition. TurfNets are fully self-contained and autonomous, even down to the type of addressing and/or the routing protocols they can use. This provides great flexibility for integration and composability of TurfNets. Clear administrative boundaries with minimal, but well-defined, control interfaces provide the basis for flexibility.

Mobility. Mobility support is another important criterion for network architectures, because more Internet nodes and subnetworks are expected to become mobile in the future.

Due to the information-hiding capabilities of TurfNet, many TurfNodes may not be directly addressable. In this event, correspondent nodes will have to address the mobile node through its external proxy addresses. Because this proxy address may not change when the mobile device moves between different TurfNets, mobility management can be a local operation that is transparent to the correspondents. In case of a Turf-local handoff, the mobile node has to merely inform the local NAT gateway about its new internal address. The hierarchical structure of composed TurfNets allows such "local" handoffs at any level in the hierarchy. For example, if a mobile node moves between TurfNets that have a common parent TurfNet, the handoff only affects the parent TurfNet. This local handoff at the parent-level is completely transparent to those correspondent nodes that are located above its parent TurfNet or in any other sub-branch of the TurfNet hierarchy. Only correspondent nodes in the same branch of the hierarchy are affected by the move. To "repair" inter-Turf communication between those correspondent nodes and the mobile node after a handoff, the parent TurfNet (where the change of inter-Turf routing to the mobile takes pace) signals the respective gateways along the path to update the relevant relaying state. Note that the full specification of TurfNet mobility management procedure is currently still under investigation and therefore not yet included here.

\section{Related Work}

This section discusses related work that also aims at resolving problems of today's Internet architecture.

TRIAD [4] is a recently proposed Internet architecture that tries to resolve the lack of end-to-end connectivity of today's NAT'ed networks by means of an explicit content layer. Similar to the TurfNet architecture, TRIAD uses name identifiers rather than addresses for node identification and routing. Because network addresses in both architectures have no end-to-end significance (they are merely used as transient routing tags), both approaches rely on name lookup mechanisms to find and setup the high-level routing path across the independent network domains. However, the main difference between TRIAD and TurfNet lies in the way they handle high-level routing. Whereas TRIAD uses source-routing to forward packets, TurfNet uses the name registration and lookup mechanisms to configure high-level routing paths and their necessary address/protocol translation state. Another major difference is that TRIAD fully relies on IPv4 support in all transient network domains, whereas TurfNet can mask diverse addressing schemes and network protocols in transient network domains through the concept of proxy addresses and protocol translation capabilities at the gateway nodes. 
Plutarch [3] is another internetworking architecture that aims to subsume existing architectures like the Internet. Similar to TurfNet, the aim of Plutarch is to make the heterogeneity of existing and future networks explicit. To translate communication among heterogeneous network environments (contexts), Plutarch introduces the concepts of interstitial functions, which allow data to pass between two adjoining contexts. Nevertheless, Plutarch differs fundamentally from the TurfNet architecture with respect to naming and routing. Firstly, it assumes different namespaces per context, and secondly, routing is based on sender selection of a context chain and the configuration of the required interstitial functions in gateway nodes along the context chain.

The NAT-extended IP architecture IPNL [5] is another closely related approach. Like the TurfNet architecture, it also aims at truly isolating administratively independent IP subnetworks and domains by providing a mechanism to loosely integrate them. The proposed idea also uses NAT middleboxes to integrate networks with a potentially overlapping address space in a way that does not require renumbering. Two fundamental differences to the TurfNet architecture exist. First, it does not limit the number of hierarchical composition steps, whereas IPNL considers at the most two levels: NAT'ed private realms local networks and global middle realms networks. Second, the TurfNet architecture does not depend on specific addressing schemes and network protocols, but rather tries to provide a general solution that can integrate many approaches.

A technique similar to IPNL is proposed in 4+4 [19]. Here, address translation occurs also between private and public realms, although in this case it is envisaged to support several "middle" realms. In comparison to IPNL, 4+4 is simpler and allows incremental deployment in today's networks. A fundamental difference between the TurfNet architecture and IPNL and 4+4 is that both architectures rely on globally unique host addresses, which consists of the concatenation of the nodes public/global and private/local addresses.

Another related work is the Address Virtualization Enabling Service (AVES) [20]. The key idea here is to virtualize non-IP hosts or hosts that are not globally routable through so called waypoints. The waypoints then act as relays between standard IP hosts and those typically not addressable/reachable hosts. In that sense, AVES is very specific as it only tries to provide bi-directional connectivity for individual hosts. The real overlap between TurfNet and AVES lies in the way the waypoint relays are selected. Similar to TurfNet, non-IP hosts are dynamically bound to waypoints during the name resolution in a connection-initiator-specific fashion.

The concept of 'network pointers' proposed for SelNet [21] is another related approach. Instead of using standard network addresses within data packets, SelNet introduces so called selectors, which allow the network pointers (packet handlers) to change the processing semantics of packets as they traverse the network. As a result, SelNet requires a specialized routing protocol that allows mapping of routing information onto selectors.

This section has shown that TurfNet is in many aspects related to recent architectural proposals. However, the ability to compose completely diverse autonomous networks and the resultant benefits are a fundamentally new feature of the TurfNet architecture. The fact that TurfNets can completely mask fundamental difference within individual network domains is especially important in the light of growing tussles in cyberspace [1]. 


\section{Conclusion and Future Work}

This paper introduces the TurfNet architecture for global, packet-switched internetworks. The architecture addresses the challenges of deploying networks in competitive environments that require means for autonomous control and information hiding.

The TurfNet architecture supports horizontal and vertical composition. Vertical composition preserves full autonomy of composed TurfNets and supports integration of heterogeneous packet-based networks that use different, non-compatible networklevel protocols.

The use of a high-level control interface that only requires a common data format across all autonomous network domains allows control of border gateways that perform the required protocol and address translation for communication across TurfNet boundaries.

The autonomy and flexibility provided by TurfNets requires the use of soft state. The paper outlines methods that assure a high scalability of the architecture. An important aspect of the TurfNet architecture is the high-level or inter-Turf routing. This paper discussed the feasibility and realization of inter-Turf communication (i.e., packet processing and forwarding), but did not yet specify a specific routing mechanism. Consequently, one area of future work lies in inter-Turf routing mechanisms that account for dynamic composition of (moving) networks, as well as performance and reliability for individual end-to-end communication.

Another aspect of the TurfNet architecture that requires further consideration is the introduction of virtual "overlay" TurfNets. Such virtual Turfs can integrate servicespecific functionality into the network without complicating its basic functionality.

Finally, another focus of future work lies in evaluating the TurfNet architecture through simulations of its scalability properties; especially for large, composed networks. One important aspect of this work is the implementation and measurements of specific prefix-based address translation mechanisms.

\section{Acknowledgements}

This document is a byproduct of the Ambient Networks project, partially funded by the European Commission under its Sixth Framework Programme. It is provided "as is" and without any express or implied warranties, including, without limitation, the implied warranties of fitness for a particular purpose. The views and conclusions contained herein are those of the authors and should not be interpreted as necessarily representing the official policies or endorsements, either expressed or implied, of the Ambient Networks project or the European Commission.

\section{References}

1. D. Clark, J. Wroclawski, K. R. Sollins and R. Braden. Tussle in Cyberspace: Defining Tomorrow's Internet. Proc. ACM SIGCOMM 2002, Pittsburgh, August 2002, pp. 347-356.

2. D. Clark, R. Braden, A. Falk and V. Pingali. FARA: Reorganizing the Addressing Architecture. Proc. ACM SIGCOMM Workshop on Future Directions in Network Architecture, Germany, August 2003, pp. 313-321. 
3. J. Crowcroft, S. Hand, R. Mortier, T. Roscoe and A. Warfield. Plutarch: An Argument for Network Pluralism. Proc. ACM SIGCOMM Workshop on Future Directions in Network Architecture, Germany, August 2003, pp. 258-266.

4. D. R. Cheriton and M. Gritter. TRIAD: A Scalable Deployable NAT-based Internet Architecture. Stanford Computer Science Technical Report, January 2000.

5. P. Francis and R. Gummadi. IPNL: A NAT-Extended Internet Architecture. Proc. ACM SIGCOMM, San Diego, CA, USA, August 2001, pp.69-80.

6. K. R. Sollins. Designing for Scale and Differentiation. Proc. ACM SIGCOMM Workshop on Future Directions in Network Architecture, Germany, August 2003, pp. 267-276.

7. P. Mockapetris. Domain Names - Concepts and Facilities. RFC 1034, November 1987.

8. R. Moskowitz and P. Nikander. Host Identity Protocol Architecture. Work in Progress (draft-moskowitz-hip-arch-06.txt), June 2004.

9. H. Balakrishnan, K. Lakshminarayanan, S. Ratnasamy, S. Shenker, I. Stoica and M. Walfish. A Layered Naming Architecture for the Internet. To appear Proc. ACM SIGCOMM, Portland, OR, USA, August 2004.

10. R. Braden, D. Clark, S. Shenker and J. Wroclawski. Developing a Next-Generation Internet Architecture, July 2000. Whitepaper, available at http://www.isi.edu/newarch/ DOCUMENTS/WhitePaper.ps.

11. N. Niebert, A. Schieder, H. Abramowicz, G. Malmgren, J. Sachs, U. Horn, C. Prehofer and H. Karl. Ambient Networks - An Architecture for Communication Networks Beyond 3G. IEEE Wireless Communications, Vol. 11, No. 2, April 2004, pp.14-22.

12. C. Kappler, P. Mendes, C. Prehofer, P. Pöyhönen and D. Zhou. A Framework for Selforganized Network Composition. Proc. $1^{\text {st }}$ International Workshop on Autonomic Communication (WAC 2004), Berlin, Germany, October 2004.

13. R. Droms. Dynamic Host Configuration Protocol. RFC 2131, March 1997.

14. S. Deering and R. Hinden. Internet Protocol, Version 6 (IPv6) Specification. RFC 2460, December 1998.

15. P. Srisuresh and M. Holdrege. IP Network Address Translator (NAT) Terminology and Considerations. RFC 2663, August 1999.

16. I. Stoica_, R. Morris, D. Karger, M. Frans Kaashoek, H. Balakrishnan. Chord: A Scalable Peer-to-peer Lookup Service for Internet Applications. IEEE/ACM Transactions on Networking, Vol. 11, No. 1, February 2003, pp. 17-32.

17. C. Dubnicki, C. Ungureanu and W. Kilian. FPN: A Distributed Hash Table for Commercial Applications. Proc. HPDC-13, Honolulu, HI, USA, June 2004.

18. M. F. Kaashoek and D. R. Karger. Koorde: A simple degree-optimal distributed hash table. Proc. $2^{\text {nd }}$ Workshop on Peer-to-Peer Systems, Berkeley, CA, February 2003, pp. 98-107.

19. Z. Turanyi, A. Valko and A. Campbell. 4+4: An Architecture for Evolving the Internet Address Space Back Towards Transparency. ACM SIGCOMM Computer Communication Review, Vol. 33, No 5, October 2003, pp 43-54.

20. T.S.E. Ng, I. Stoica and H. Zhang. A Waypoint Service Approach to Connect Heterogeneous Internet Address Spaces. In Proc. of USENIX Annual Technical Conference, Boston, MA, USA, June 2001, pp. 319-332.

21. Christian Tschudin and Richard Gold. Network Pointers. Proc. $1^{\text {st }}$ Workshop on Hot Topics in Networks (HotNets-I), Princeton, New Jersey, October 2002. 